\author{
ROLAND HEGEDÜS* \\ University of Debrecen \\ hegedusroland1989@gmail.com \\ ORCID: 0000-0002-6576-5077 \\ KRISZTINA SEBESTYÉN \\ University of Nyíregyháza \\ kriszti.se@gmail.com \\ ORCID: 0000-0002-0253-0561
}

\title{
Eine Fallstudie über die regionalen Unterschiede der ungarischen Schülerleistungen
}

\section{A case study on regional differences in Hungarian pupils' school achievement}

\begin{abstract}
The sociocultural and socioeconomic background of pupils is a determinant for their learning process and it has an effect on their learning success and on how easily pupils are able to master the requirements set by school curricula and how they perform on different measuring tasks. This is the reason why we compare in our paper the reading and mathematics results of the National Competence Measurement 2013 with the English and German language results of the secondary school-leaving exams in foreign languages regarding the same pupils. We analyse the data using the SPSS program and we present the results using the MapInfo program. We examine the effects of pupils' social background, the interactions of different subjects and their regional projections as well. The pupils' achievements show the same pattern as the development patterns of different geographical areas in Hungary.
\end{abstract}

KEYWORDS: National Competence Measurement in Hungary, results of the secondary schoolleaving exam, family background, regionality, Hungary.

SCHLÜSSELWORTE: Nationalkompetenzmessung in Ungarn, Ergebnisse des Abiturs, Familienhintergrund, Regionalität, Ungarn.

* Während der Bearbeitung des Beitrags hat Roland Hegedûs ein Stipendium „Nemzeti Tehetség Program Nemzet Fiatal Tehetségeiért Ösztöndíj" (NTP-NFTÖ-18-B-250) bekommen. 


\section{EINLEITUNG}

In einem Land existieren oft geografische und regionale Unterschiede z. B. in Bezug auf den wirtschaftlichen, arbeitsmarktlichen beziehungsweise sozioökonomischen Status der in einem Gebiet lebenden Bevölkerung. In unserem Beitrag untersuchen wir die territorialen Unterschiede von Schülerleistungen im Kontext ihrer Bildung und ihres sozioökonomischen Status. Mit den internationalen Erhebungen von schulischen Leistungen nahm der Bedarf zu, die Umstände in den einzelnen Ländern zu untersuchen. Deshalb sollen in der vorliegenden Arbeit die regionalen Unterschiede der ungarischen Schülerleistungen sowie mögliche Auswirkungen auf die Wirtschaft dargestellt werden. In unserem Aufsatz analysieren wir die Muttersprache (ungarisches Leseverstehen) und die Mathematik betreffenden Ergebnisse einer der PISA-Studie ähnlichen, ungarischen Kompetenzmessung beziehungsweise die Ergebnisse des Sprachabiturs (Deutsch und Englisch). Die ungarische Kompetenzmessung ist eine longitudinale Untersuchung, die in jedem Schuljahr mit allen Schülerinnen und Schülern der sechsten, achten und zehnten Klassen so durchgeführt wird, dass die Ergebnisse später miteinander verglichen werden können. In unserer Untersuchung analysieren wir die Ergebnisse der Kompetenzmessung von Schülerinnen und Schülern des ganzen Landes, die 2013 die zehnte Klasse besuchten. Die Muttersprache und Mathematik bilden die Grundlage vieler Fächer. Fremdsprachenkenntnisse sind heutzutage unerlässliche Kompetenzen in Bezug auf die Weiterbildung und spätere Chancen auf dem Arbeitsmarkt.

Man kann in Ungarn bedeutende Entwicklungsunterschiede z. B. zwischen den vom Plattensee nördlich liegenden (entwickelten) Gebieten und dem (eher rückständigen) nordöstlichen Teil des Landes feststellen. Deshalb ist es wichtig zu wissen, was für Schülerleistungen auf den einzelnen Gebieten von Ungarn aufzuweisen sind und welche Zusammenhänge zwischen den Schülerleistungen und dem soziökonomischen Status der Familien bestehen. Auch die OECD vertritt den Standpunkt, dass Bildung ein Mittel sein kann, mit dessen Hilfe die Benachteiligten aus ihrer Situation ausbrechen können (Polónyi 2013).

\section{GESELLSCHAFTLICHER STATUS UND FREMDSPRACHENKENNTNISSE}

Heutzutage wird die gesellschaftliche Rolle einer Person immer häufiger durch die schulische Bildung und nicht durch den Familienhintergrund bestimmt. Dieser Prozess verhält sich proportional zur gesellschaftlichen Ent- 
wicklung (Fehérvári 2015: 190-191). Das Schulsystem konserviert die gesellschaftlichen sowie die beruflichen Gruppen (Bourdieu 1983: 185; Petneki 2002; Gogolin 2014: 410), was im Prinzip bedeutet, dass die ärmeren Schülerinnen und Schüler keine Chance von der Schule bekommen, eine höhere gesellschaftliche Position zu erreichen. Die familiäre Umgebung, der Bildungsstand der Eltern sowie der sozioökonomische Status der Familie haben eine bedeutende Wirkung auf die Schulleistungen (Coleman et al. 1966; Ettrich, Krause \& Hofer 1996; Niedrig 2002: 4; Oswald \& Krappman 2004: 752-753; Scheiner 2006; Rolff et al. 2008). Die zu Hause und die in der Schule benutzten Sprachen stehen zum Beispiel im Falle der Schülerinnen und Schüler mit höherem gesellschaftlichem Status zueinander sehr nahe, weshalb sie keine sprachlichen Nachteile haben und die Schule erfolgreich abschlieBen (Bernstein 2003: 14, 58-63; Entorf 2005: 134-135; Gogolin 2014: 410, 419). Bessere Leistungen sind im Leseverstehen sichtbar, aber auch in anderen Fächern, weil gutes Leseverstehen in den meisten Fächern zu einem erfolgreichen Abschluss benötigt wird (Gogolin 2014: 424). Man kann bei der Untersuchung von Zusammenhängen zwischen dem Leseverstehen und der Mathematik (Garami 2009a), den muttersprachlichen und den fremdsprachlichen Kenntnissen (Kaikkonen 1993; Hesse, Göbel \& Hartig 2008) sowie den fremdsprachlichen und den mathematischen Leistungen (Szirmai 2003) feststellen, dass eine positive Wechselwirkung unter all diesen Faktoren besteht. Das mathematische Denken ist zum Beispiel mit der Effektivität des Fremdsprachenlernens verknüpft, denken wir nur an die Logik, die Linguistik und die grammatischen Zusammenhänge.

Der gesellschaftliche Status der Schülerinnen und Schüler kann für die Leistungen entscheidend sein. Dies gilt auch für die Fremdsprachenkenntnisse, weil Schülerinnen und Schüler mit höherem gesellschaftlichem Status meistens (auch) Englisch lernen (Csapó 2001: 30; Nikolov 2003: 63; Nikolov \& Vígh 2012: 263). Die deutsche Sprache wird in erster Linie von Schülerinnen und Schülern mit niedrigerem gesellschaftlichen Status gewählt beziehungsweise von denjenigen, die diese Sprache in der Zukunft in ihrem späteren Beruf (z. B. in technischen Bereichen) brauchen. Natürlich wird die Wahl einer Sprache auch durch das jeweilige Sprachangebot der Schulen selbst beeinflusst. Anhand der Angaben des Bildungsstatistischen Jahrbuches (Csécsiné Máriás, Hagymássy \& Könyvesi 2015) konnte festgestellt werden, dass das Deutsche sowie das Englische die zwei populärsten Sprachen in den untersuchten Jahren waren.

Der Bildungserfolg der Schülerinnen und Schüler kann neben dem gesellschaftlichen Status der Familie auch durch die geografischen und regionalen Gegebenheiten beeinflusst werden (vgl. Fürst \& Schubert 2001; Linberg \& Wenz 2017). Dies gilt sowohl im Fall der Weiterbildungsmotivation 
an einer Mittel- sowie einer Hochschule, als auch später im Fall der Bewerbung um einen Arbeitsplatz (Veroszta 2010; Fekete, Hegedús \& Sebestyén 2016). Auch der finanzielle Status der Familie kann die Wahl der Bildungsinstitution bestimmen. Schülerinnen und Schüler beziehungsweise Studentinnen und Studenten mit niedrigerem gesellschaftlichem Status können keine ferner liegende Bildungsinstitution wählen, weshalb sie die näher liegenden Institutionen bevorzugen (Schultz 1998; Denzler \& Wolter 2010: 10-15; Fekete et al. 2016: 113). Diese Situation impliziert auch, dass die Personen in ihrer Region bleiben werden.

Wenn die Schülerinnen und Schüler am Ende ihrer Bildungslaufbahn an ihrem Wohnort oder in der Nähe des Wohnortes einen Arbeitsplatz finden möchten, ist es wichtig zu wissen, welche Gebiete in Ungarn entwickelt und welche weniger entwickelt sind. In dieser Hinsicht sind die herausragenden Gebiete die Hauptstadt Budapest und ihre Umgebung. Diesen folgen die von der Hauptstadt nordwestlich liegenden, von der Industrie geprägten Gebiete, der mittlere Teil Ungarns sowie die Komitatssitze. Das nordöstliche Gebiet des Landes und Südtransdanubien haben die größten Nachteile, besonders die Gebiete in Grenznähe (Kozma 1996: 253-256; Süli-Zakar 2003; Baranyi, Kanalas \& Kiss 2006).

Im vorliegenden Aufsatz untersuchen wir zwei Dimensionen der Bildungseffektivität: gesellschaftliche und regionale Variablen. Bei diesen zwei Dimensionen geht es um die Ergebnisse der nationalen Kompetenzmessung und die des fremdsprachlichen Abiturs in der Mittelstufe. Im ungarischen Schulsystem können die Schülerinnen und Schüler an Gymnasien ${ }^{1}$ und Fachmittelschulen ${ }^{2}$ ein Abitur ablegen (vgl. Óhidy 2007). Alle Schülerinnen und Schüler müssen in der Mittelstufe in fünf Fächern - Ungarisch, Mathe-

${ }^{1}$ Die Gymnasien sind vier-, sechs- oder achtjährig. Die vertikale Differenzierung begann bereits vor 1990, und nach der Wende erfuhr sie eine wesentliche Beschleunigung. Alle drei Arten von Gymnasien bereiten die Schüler auf das Abitur und - ausgesprochen oder unausgesprochen - auf ein Hochschulstudium, allerdings nach unterschiedlichen Lehrplänen vor (vgl. Óhidy 2007: 87).

2 Die Fachmittelschule bietet sowohl ein Abitur als auch einen berufspraktischen Abschluss in vier bzw. fünf Jahren. In den ersten vier Jahren werden „praktische berufliche Basiskenntnisse gleichzeitig mit einer fundierten Allgemeinbildung“ (Fuchs 2001: 39) vermittelt. Im Anschluss daran werden die Absolventen in einem weiteren Jahr auf den Abschluss eines anerkannten Ausbildungsberufs vorbereitet (Fuchs 2001). Das Abschlussexamen unterscheidet sich vom dem der Gymnasien dadurch, dass es auch einen berufspraktischen Teil enthält und bei der Prüfung nicht nur ein Lehrer und ein Beauftragter des Bildungsministeriums, sondern auch je ein Vertreter der Unternehmerschaft und der örtlichen Handelskammer anwesend sind. Das Abschlusszeugnis berechtigt einerseits zum Hochschuleintritt, andererseits gibt es einen, mit dem Facharbeiterbrief vergleichbaren Qualifikationsnachweis (Fuchs 2001). Seit 1. September 2016 nennt man diesen Schultyp ein Fachgymnasium (Gesetz LXV/2015). 
matik, Geschichte, eine Fremdsprache und in einem frei gewählten Fach das Abitur ablegen. Das zweistufige Abitursystem wurde mit der Verordnung Nr. 16/2000 (II. 17.) eingeführt und hat zwei Funktionen: Das Abitur funktioniert jetzt nicht mehr nur als eine Prüfung, die die Mittelschulausbildung abschließt, sondern es ist zugleich auch eine Aufnahmeprüfung in das Hochschulsystem. Jede Mittelschule darf das Abitur in der Mittelstufe organisieren, aber nur ausgewählte Mittelschulen dürfen die Oberstufenabiturprüfung anbieten. Die Oberstufenabiturprüfung wird nur von den Schülerinnen und Schülern gewählt, die sie zur Aufnahme eines Hochschulstudiums brauchen (Einhorn 2007: 81). Das zweistufige Abitur misst in Fremdsprachen insgesamt drei Stufen des Gemeinsamen Europäischen Referenzrahmens (GER 2002): In der Mittelstufe werden die Stufen A2 und B1, in der Oberstufe die Stufe B2 ${ }^{3}$ geprüft. Im Abitur werden die vier Sprachkompetenzen - das Leseverstehen, das Hörverstehen, das Schreiben und das Sprechen - sowie die Grammatikkenntnisse gemessen. Das Abitur können sie gemäß bestimmten Bedingungen früher oder am Ende der Bildungslaufbahn absolvieren (Verordnung Nr. 100/1997 [VI. 13.]). Die bedeutendsten Sprachen in Ungarn sind Englisch und Deutsch, weshalb die Schülerinnen und Schüler eine von diesen Sprachen für die Abiturprüfung wählen (Csécsiné Máriás et al. 2015; Földes 2016: 21-25).

\section{3. ÜBER DIE NATIONALKOMPETENZMESSUNG UND DIE REGIONALITÄT}

Die Nationalkompetenzmessung (NKM) ähnelt der PISA-Messung, weil alle Schülerinnen und Schüler bestimmter Jahrgänge an dieser Messung teilnehmen. Sie ist aber eine Eigenheit des ungarischen Bildungssystems. Wegen dieser Beziehung gehen wir auch kurz auf die PISA-Ergebnisse ein. In Ungarn ist der Einfluss des Familienhintergrundes auf die Schulbildung bedeutend höher als im PISA-Durchschnitt (Arató \& Varga 2004: 503-504). Dazu kommt noch, dass die Integration und die Inklusion bedeutend kleiner sind als in den anderen Ländern. Die Schülerleistungen werden hiervon negativ beeinflusst, wodurch die benachteiligten Schülerinnen und Schüler noch mehr ins Hintertreffen geraten (Csapó, Molnár \& Kinyó 2009), was internationale Aufsätze bestätigen (z. B. Benito, Alegre \& Gonzàlez-Balletbò 2014). Eine sehr wichtige Rolle der Schule besteht darin, gesellschaftliche Differenzen auszugleichen. In Ungarn kann man aber erfahren, dass die

\footnotetext{
${ }^{3}$ Seit 1. Januar 2017 wird in der Mittelstufe nur eine GER-Stufe, die Stufe B1 gemessen (Élő idegen nyelv vizsgakövetelmények 2016: 1).
} 
Schulen, diese Nachteile nicht kompensieren und dadurch diese Aufgabe nicht meistern können (Imre 2002: 63-64). Die Schülerleistungen werden von der gesellschaftlichen Umgebung, dem Familienhintergrund, dem Familienleben - z. B. der Beziehung mit den Eltern, den gemeinsamen Familienentscheidungen -, dem Erziehungsstil sowie der Einstellung der Lehrkräfte bedeutend beeinflusst (Oswald \& Krappmann 2004: 755; Szczesny \& Watermann 2009: 176, 189; Furcsa 2014: 14-15).

Hinsichtlich der PISA-Ergebnisse anderer Länder kann man feststellen, dass sie sich auf einer breiten Skala bewegen. In Deutschland beispielsweise gibt es auch eine Schicht von Schülerinnen und Schülern, die den vorgelegten Text nicht verstehen können, weshalb der nationale Schülerleistungsdurchschnitt bedeutend niedriger ist. Wenn die Schülerinnen und Schüler den Text nicht verstehen können, dann können sie auch nicht die dazu gehörenden Aufgaben lösen. Daher wäre es wichtig, diese Fähigkeiten und Kompetenzen schon in der Grundschule zu entwickeln und stetig zu verbessern (Artelt, Schiefele, Schneider \& Stanat 2002). Zurzeit kann man feststellen, dass die Leistungen von Schülerinnen und Schülern mit niedrigerem sozioökonomischen Status auch in Deutschland niedriger sind als die Leistungen von Schülerinnen und Schülern aus Familien mit einem höheren Status (Entorf 2005: 134-136; Neumann, Nagy, Trautwein \& Lüdtke 2009; Linberg \& Wenz 2017: 92-93).

In Verbindung mit den PISA-Ergebnissen kann noch ein anderes Problem festgestellt werden. Dieses tritt in den USA auf, wo 20 Millionen Einwandererinnen und Einwanderer leben und deren Kinder die englische Sprache nicht so gut beherrschen. Diese Schülerinnen und Schüler zeigen schwächere Leistungen in den verschiedenen Tests. Bei den Schülerinnen und Schülern in den USA zeigt sich auch ein Unterschied zwischen zwei Schichten, Arm und Reich. Die Schülerinnen und Schüler, deren gesellschaftlicher Status es ermöglicht, bekommen Nachhilfeunterricht, so dass sie bessere Leistungen in den englischen Tests erzielen können. Währenddessen erreichen die ärmeren Schülerinnen und Schüler ohne solche Privatstunden niedrigere Werte in den Tests. Die Probleme des Leseverstehens wirken sich auch auf die Leistungen im Fach Mathematik aus. Diese Ergebnisse beschränken auch die Teilnahme der benachteiligten Schülerinnen und Schüler am Studium an den Hochschulen. Die Hochschulbildung wäre aber sehr wichtig, weil 63 Prozent der Arbeitsplätze im Jahre 2018 in den USA an einen Hochschulabschluss geknüpft sind. Nach Meinung von Experten sollte die Arbeit der Lehrer verbessert werden und die Lehrkräfte sollten die Schülerinnen und Schüler zur Hochschulbildung motivieren (Henry, Baltes \& Nistor 2015: 94-96). 
Im Allgemeinen kann man feststellen, dass die Schülerleistungen in Mathematik und in der Muttersprache bei denjenigen Schülerinnen und Schülern besser sind, deren Eltern zu Hause eine gemeinsame Sprache sprechen und die keinen Migrationshintergrund haben. Neben den oben genannten Faktoren - der höheren sozialen Position und der besseren finanziellen Situation der Herkunftsfamilie - tragen diese Faktoren ebenfalls dazu bei, dass diese Schülerinnen und Schüler an Privatstunden einfacher teilnehmen können (Rolff et al. 2008: 293-294) und so ihre Leistungen verbessern können. Je höher der Bildungsstand der Eltern ist, desto besser sind die muttersprachlichen Leistungen der Schülerinnen und Schüler.

Die Ergebnisse der Nationalkompetenzmessung hängen, wie bereits erwähnt, auch eng mit den regionalen Unterschieden in Ungarn zusammen. Das bedeutet, dass die Kompetenzergebnisse im westlichen Teil des Landes, in den Gebieten nahe der österreichischen Grenze und in der Hauptstadt bedeutend höher sind als im überregionalen Durchschnitt. Diese Gebiete kann man als dynamische Fokuspunkte bezeichnen. Dazu gehören auch die Landkreise der Komitatssitze in den nicht so entwickelten Gebieten (Garami 2009b: 245-246). In den entwickelten Gebieten ist die Abweichung auch viel kleiner als da, wo die gesellschaftliche und die ökonomische Entwicklung niedriger ist. Nur diejenigen Gebiete können als nicht benachteiligt angesehen werden, die eine lange Bildungsgeschichte haben (Garami 2014: 425-429).

Die regionalen Unterschiede in der Nationalkompetenzmessung und der Bildungseffektivität zeigen eindeutig, dass höhere Schülerleistungen mit einer entwickelten Ökonomie einhergehen. So kann man in den vom Plattensee nördlich liegenden Gebieten, in der Hauptstadt und auch im Komitat Békés beobachten, dass es hier eine entwickelte Ökonomie und höhere Kompetenzergebnisse gibt. Diese Zusammenhänge wirken sich auch auf die Zukunft aus: Wenn die Schülerleistungen in einem Gebiet höher sind, dann wird sich eine Schülerin oder ein Schüler wahrscheinlicher um einen Studienplatz an einer Hochschule bewerben und wird zum Studium in einem landesweit anerkannten Fach aufgenommen (Hegedús 2016: 270-275).

In einer ungarischen Untersuchung wurden die Schulen anhand mehrerer Variablen in zwei große Gruppen eingeteilt. Die eine Gruppe bilden die resilienten Schulen, die Nachteile haben, aber gute Ergebnisse aufweisen können. Zur anderen Gruppe gehören die gefährdeten Schulen, die schlechte Ergebnisse haben. Man kann einen großen Anteil der guten Schulen in der Hauptstadt und im Komitat Békés finden. Diese Schulen können durch innovative Lehrkräfte charakterisiert werden, die ihre Schülerinnen und Schüler zu besseren Leistungen motivieren. Der größte Anteil der gefährdeten Schulen liegt im Norden Ungarns, der als ein benachteiligtes Gebiet be- 
zeichnet werden kann. Hier können die Lehrerinnen und die Lehrer die Schülerinnen und Schüler nicht zu besseren Leistungen motivieren (Széll 2015).

Man kann auch anhand der Schultypen bedeutende Unterschiede bemerken. Die Schülerinnen und Schüler in den sechs- sowie achtklassigen Gymnasien haben immer die besten Ergebnisse, und die Schülerinnen und Schüler in den Fachschulen 4 haben die schlechtesten Leistungen (Balázsi \& Horváth 2010: 345-346). Man kann aber feststellen, dass auch die schwächeren Schülerinnen und Schüler in einer über bessere Durchschnittsleistungen verfügenden Schule bessere Leistungen erreichen können, als man es von diesen Schülerinnen und Schülern erwarten würde (Pop-Eleches \& Urquiola 2013: 1322-1323).

\section{METHODOLOGIE}

\subsection{Die untersuchte Gruppe}

Wir analysierten zwei Datenbanken, deren Daten vom ungarischen Bildungsamt stammen. Einerseits untersuchten wir die Datenbank der Nationalkompetenzmessung 2013, andererseits die Datenbank des Abiturs zwischen den Jahren 2013 und 2015. Die Nationalkompetenzmessung wird jeden Frühling im ganzen Land unter allen Schülerinnen und Schülern in den sechsten, achten und zehnten Klassen durchgeführt. Wir beschäftigen uns hier nur mit den Ergebnissen der zehnten Klassen, weil diese Schülerinnen und Schüler nur wenige Jahre vor dem Abitur stehen. In dieser Datenbank kann man die Daten von mehr als 90000 Schülerinnen und Schülern finden. Unter denen sind auch solche, die eine Fachschule besuchen, aber am Ende ihrer Bildungslaufbahn kein Abitur ablegen können, und auf deren Untersuchung wir deshalb in diesem Aufsatz verzichten. Ferner beschäftigen wir uns nicht mit den Schülerinnen und Schülern, über die im Index keine Auskunft zum Familienhintergrund ${ }^{5}$ und keine Ergebnisse im Leseverstehen beziehungsweise in der Mathematik vorhanden waren. So schließlich bekamen wir eine Datenbank mit den Angaben von 55156 Schülerinnen und Schülern. In Tabelle 1 kann man den Frauen-Männer-Anteil sowie den Schüleranteil in den verschiedenen Schultypen sehen.

${ }^{4}$ Die Fachschulen bieten eine zweijährige Ausbildung in einer Mischung aus Schule, Lehre und handwerklicher Ausbildung an (vgl. Óhidy 2007: 88). Seit 1. September 2016 nennt man diesen Schultyp eine Fachmittelschule (Gesetz LXV/2015).

${ }^{5}$ Der Familienhintergrundindex enthält die folgenden Angaben: den Bildungsstand der Mutter sowie des Vaters, die Zahl der Computer, die Zahl der Bücher und die Zahl der eigenen Bücher. 
Tabelle 1. Grunddaten der Datenbanken

\begin{tabular}{|l|c|c|}
\hline \multicolumn{1}{|c|}{ Veränderlichen } & NKM 2013 & Abitur 2013-2015 \\
\hline Männer & $25920(47,0 \%)$ & $24182(48,6 \%)$ \\
\hline Frauen & $29236(53,0 \%)$ & $25550(51,4 \%)$ \\
\hline Gymnasium & $28437(51,6 \%)$ & $26115(52,5 \%)$ \\
\hline Fachmittelschule & $26719(48,4 \%)$ & $23617(47,5 \%)$ \\
\hline Insgesamt & 55156 & 49732 \\
\hline
\end{tabular}

Datenquellen: NKM 2013; Abitur 2013-2015

In der Tabelle 1 sind auch die Angaben der Datenbank des Abiturs 2013-2015 zu finden. Diese Daten wurden von der Webseite des ungarischen Bildungsamts (Kétszintú 2016) in mehreren Teilen heruntergeladen. Anschließend wurden alle Angaben in der Datenbank zusammengeführt. In dieser Datenbank befinden sich die Angaben von Schülerinnen und Schülern, die in der Regelschulzeit ${ }^{6}$ oder früher das Abitur in der Mittelstufe in Deutsch oder in Englisch ablegten. Wir können in dieser Datenbank ganz ähnliche Anteile der Schülerinnen und Schüler wie in der Nationalkompetenzmessung feststellen: Es gibt in der Datenbank „Abitur 2013-2015“ auch mehr Frauen, beziehungsweise mehr Gymnasialschülerinnen und Gymnasialschüler. Zwischen den beiden Datenbanken besteht der Zusammenhang, dass die Schülerinnen und Schüler, die 2013 an einer Kompetenzmessung in der zehnten Klasse teilgenommen haben, zwischen 2013 und 2015 früher oder am Ende der Regelschulzeit das Sprachabitur ablegen konnten. Wir untersuchten nur die Schülerinnen und Schüler, die an beiden Messungen an der Kompetenzmessung und am Abitur - teilgenommen haben.

\subsection{Die Methoden}

Die Gemeinsamkeit der beiden Datenbanken liegt in der Regionalität, da die Landkreise anhand der Ergebnisse in Mathematik, im Leseverstehen und im Abitur, des Familienhintergrundes sowie des Anteils der Schülerinnen und Schüler in den Gymnasien charakterisiert werden. Im ersten Schritt rechneten wir den Durchschnitt der Landkreise aus, dann setzten wir diese Daten in eine neue Tabelle. Wir machten eine Verteilungsuntersuchung, so

${ }^{6}$ Das Abitur kann man in einem Fach nicht nur am Ende der Mittelschule ablegen, sondern auch früher, wenn man bestimmte Bedingungen erfüllt. 
dass wir anhand der Ergebnisse feststellen konnten, dass unsere Variablen keine Normalverteilung haben. Wir benutzten die Spearman-Korrelation? um die Zusammenhänge der Variablen zu untersuchen.

Im nächsten Schritt analysierten wir die Unterschiede in den verschiedenen Regionen in Bezug auf die Schülerleistungen, den Familienhintergrund und den Anteil der Gymnasialschülerinnen sowie Gymnasialschüler. Mit einer Clusteranalyse gruppierten wir die Landkreise und bekamen fünf Cluster als Ergebnis. Mit dem MapInfo-Programm skizzierten wir die Ergebnisse auf einer Landkarte, so dass die regionalen Unterschiede sichtbar wurden. Es gibt einige Landkreise, in denen keine Gymnasien und / oder Fachmittelschulen zu finden sind, und die wir deshalb weiß markierten.

Schließlich untersuchten wir, welche Unterschiede hinsichtlich der Schülerleistungen und der gesellschaftlichen Position zu erkennen sind. Wir arbeiteten mit den Durchschnittswerten der Datenbanken, so dass wir die Angaben miteinander vergleichen konnten. $\mathrm{Zu}$ dieser Untersuchung machten wir eine Varianzanalyse.

\section{DISKUSSION DER ERGEBNISSE}

Zuerst analysierten wir, welche Zusammenhänge es unter den nach den Landkreisen aggregierten Variablen gibt. In der Untersuchung zeigte der Familienhintergrundindex eine starke Korrelation mit allen Variablen. Er steht im stärksten Zusammenhang mit dem Leseverstehen (0.788) und im schwächsten mit dem Deutsch-Abitur in der Mittelstufe (0.616), obwohl auch dieser letzte Zusammenhang bedeutend ist. Die Ergebnisse in Mathematik zeigen sowohl mit dem Leseverstehen (0.932), als auch mit dem Abitur in der Mittelstufe in Englisch (0.760) und in Deutsch (0.663) einen sehr starken Zusammenhang. Es ist interessant, dass die Korrelation zwischen den Ergebnissen in Mathematik und in Deutsch um eine Zehntel kleiner ist. Das Leseverstehen zeigt mit den Leistungen im Abitur einen stärkeren $\mathrm{Zu}-$ sammenhang als mit den Ergebnissen in Mathematik. Die Korrelation des Leseverstehens mit Englisch ist 0.791, mit Deutsch 0.722. Zwischen den Ergebnissen in Englisch und Deutsch gibt es auch einen starken Zusammenhang (0.780). Diese letzteren Angaben zeigen auch: Je höher die Ergebnisse in einem Landkreis in Englisch sind, desto höher sind die Ergebnisse in Deutsch (Tabelle 2).

7 Bei der Datenverteilung kann man den Kolmogorov-Smirnov-Test (bei einer Normalverteilung) oder die Spearman-Korrelation (mangels einer Normalverteilung) benutzen. 
Tabelle 2. Die Ergebnisse der Korrelationsanalyse unter den Variablen

\begin{tabular}{|l|c|c|c|c|c|}
\hline \multicolumn{1}{|c|}{ Veränderlichen } & $\begin{array}{c}\text { Familien- } \\
\text { hintergrund- } \\
\text { index }\end{array}$ & $\begin{array}{c}\text { Ergebnisse } \\
\text { in der } \\
\text { Mathematik }\end{array}$ & Ergebnisse im & $\begin{array}{c}\text { Abitur in } \\
\text { der Mittel- } \\
\text { stufe in } \\
\text { Englisch }\end{array}$ & $\begin{array}{c}\text { Abitur in der } \\
\text { Mittelstufe } \\
\text { in Deutsch }\end{array}$ \\
\hline $\begin{array}{l}\text { Familienhintergrund- } \\
\text { index }\end{array}$ & 1 & $0,745^{* *}$ & $0,788^{* *}$ & $0,686^{* *}$ & $0,616^{* *}$ \\
\hline $\begin{array}{l}\text { Ergebnisse in der } \\
\text { Mathematik }\end{array}$ & $0,745^{* *}$ & 1 & $0,932^{* *}$ & $0,760^{* *}$ & $0,663^{* *}$ \\
\hline $\begin{array}{l}\text { Ergebnisse im Lese- } \\
\text { verstehen }\end{array}$ & $0,788^{* *}$ & $0,932^{* *}$ & 1 & $0,791^{* *}$ & $0,722^{* *}$ \\
\hline $\begin{array}{l}\text { Abitur in der Mittel- } \\
\text { stufe in Englisch }\end{array}$ & $0,686^{* *}$ & $0,760^{* *}$ & $0,791^{* *}$ & 1 & $0,780^{* *}$ \\
\hline $\begin{array}{l}\text { Abitur in der Mittel- } \\
\text { stufe in Deutsch }\end{array}$ & $0,616^{* *}$ & $0,663^{* *}$ & $0,722^{* *}$ & $0,780^{* *}$ & 1 \\
\hline
\end{tabular}

$(\mathrm{NNKM}=55156, \mathrm{NA}=49732){ }^{* *}: \mathrm{p}<0.001$ Datenquellen: NKM 2013; Abitur 2013-2015

Die Prüfung im Leseverstehen wird auf Ungarisch geschrieben, also meistens in der Muttersprache der Schülerinnen und Schüler. Das bedeutet, dass diejenigen Schülerinnen und Schüler, die gute Leistungen im Leseverstehen aufweisen, anhand der Korrelation auch gute Ergebnisse in den Fremdsprachen und in der Mathematik haben. So wird die Theorie unterstützt, dass die Gewandtheit in der Muttersprache auch im Fall anderer Fächer bessere Ergebnisse ergibt (Gogolin 2014: 424). Man kann dies nicht nur für die in der Muttersprache unterrichteten Fächer, sondern auch für die Fremdsprachen behaupten. Dies ist zum Beispiel in solchen Fällen wichtig, in denen die Schülerinnen und Schüler die Bedeutung eines Wortes oder einer Aussage in der Muttersprache brauchen.

Im nächsten Schritt unserer Untersuchung wurden die Landkreise anhand der früher benutzten Variablen - Mathematik, Leseverstehen und der Ergebnisse des Abiturs in der Mittelstufe in Englisch und in Deutsch - um eine weitere Variable, dem Anteil der Gymnasialschülerinnen und Gymnasialschüler in den eigenen Landkreisen, ergänzt. Die Gruppierung der Landkreise führten wir anhand dieser fünf Variablen mit Hilfe einer Clusteranalyse durch. Als Ergebnis bekamen wir fünf, gut voneinander trennbare Cluster. In dem (1) Cluster der „Rückständigen“ finden sich die Landkreise (insgesamt 14), die entlang aller Variablen die schlechtesten Ergebnisse zeigen. Der Anteil der Schülerinnen und Schüler, die die Fachmittelschule besuchen, ist hier am größten. Ein bisschen besser ist die Lage des (2) Clusters der "Zurückbleibenden" (insgesamt 21 Landkreise), weil alle Ergebnisse um ein wenig höher sind als die Ergebnisse des vorigen Clusters. Ein bedeu- 
tender Unterschied besteht darin, dass die Mehrheit der Schülerinnen und Schüler des zweiten Clusters Gymnasien besucht, aber die Gymnasien ihnen nicht helfen, bessere Ergebnisse zu erreichen. Im nächsten (3) Cluster „Unterwegs" befinden sich insgesamt 54 Landkreise. Ihre Ergebnisse stehen in allen Fällen in der Nähe des Durchschnitts, aber die Clustermittelpunkte liegen immer ein wenig unter den Durchschnittswerten der untersuchten Datenbanken. Wir benutzen den Namen "Unterwegs", weil diese Landkreise sich in der Zukunft entweder weiterentwickeln oder zurückfallen könnten. Der folgende (4) Cluster der „Fortschreitenden“ zählt 64 Landkreise, die mit ihren Ergebnissen über dem Durchschnitt stehen. Das bedeutet, dass diese Landkreise bessere Schülerleistungen und einen höheren Familienhintergrundindex aufweisen sowie dass der Anteil der Gymnasialschülerinnen und Gymnasialschüler um ein wenig höher ist. Im letzten (5) Cluster der „Entwickelten“ gibt es insgesamt 15 Landkreise, in denen die Schülerleistungen, der Familienhintergrundindex und der Anteil der Gymnasialschülerinnen und Gymnasialschüler auch bedeutend höher liegen (Tabelle 3).

Tabelle 3. Die nach den sechs Variablen gestalteten Cluster

\begin{tabular}{|l|c|c|c|c|c|}
\hline \multicolumn{1}{|c|}{ Veränderlichen } & $\begin{array}{c}\text { Zurückblei- } \\
\text { bende }\end{array}$ & Entwickelte & Unterwegs & $\begin{array}{c}\text { Fortschrei- } \\
\text { tende }\end{array}$ & Rückständige \\
\hline $\begin{array}{l}\text { Familienhintergrund- } \\
\text { index }\end{array}$ & $-0,382$ & 0,431 & $-0,067$ & 0,200 & $-0,554$ \\
\hline $\begin{array}{l}\text { Ergebnisse in der Ma- } \\
\text { thematik }\end{array}$ & $-1,026$ & 1,198 & $-0,195$ & 0,613 & $-1,838$ \\
\hline $\begin{array}{l}\text { Ergebnisse im Lesever- } \\
\text { stehen }\end{array}$ & $-1,009$ & 1,327 & $-0,214$ & 0,607 & $-1,902$ \\
\hline $\begin{array}{l}\text { Abitur in der Mittelstufe } \\
\text { in Englisch }\end{array}$ & $-0,994$ & 1,577 & $-0,280$ & 0,589 & $-1,722$ \\
\hline $\begin{array}{l}\text { Abitur in der Mittelstufe } \\
\text { in Deutsch }\end{array}$ & $-0,939$ & 1,517 & $-0,506$ & 0,613 & $-1,023$ \\
\hline $\begin{array}{l}\text { Anteil der Gymnasial- } \\
\text { schüler und Gymnasial- } \\
\text { schülerinnen }\end{array}$ & 0,903 & 1,412 & $-0,291$ & 0,010 & $-1,791$ \\
\hline Zahl der Landkreise & 21 & 15 & 54 & 64 & 14 \\
\hline
\end{tabular}

(NNKM = 55156, NA = 49732). Datenquellen: NKM 2013; Abitur 2013-2015

Danach bildeten wir diese Cluster auf einer Landkarte ab, wodurch die regionalen Umstände aller Landkreise sichtbar werden. Wir markierten die niedrigeren Leistungen mit weißen Punkten, die höheren Leistungen mit Grau und mit Schwarz. Das gestreifte Muster zeigt die Landkreise des Clus- 
ters "Unterwegs", und die weiße Farbe bezeichnet solche Landkreise, in denen keine Gymnasien oder Fachmittelschulen zu finden sind.

In der Abbildung 1 wird demnach sichtbar, dass die Mehrheit der entwickelten Landkreise zur hauptstädtischen Umgebung sowie zum nordwestlichen Teil Ungarns gehört, wie: der Landkreis Celldömölk, Szentgotthárd, Kapuvár sowie Pannonhalma. Diese Landkreise sind die am weitesten entwickelten Teile des Landes. Es gibt aber drei weitere südliche Landkreise in diesem Cluster: Sárbogárd, Tolna und Bácsalmás. Man kann außerdem feststellen, dass sich die fortschreitenden Landkreise in der Region von Westund Mitteltransdanubien konzentrieren. Hier gibt es einen oder zwei Landkreise aus dem Cluster "Unterwegs“. Dazu gehören auch der Landkreis Vasvár aus der Gruppe der „Rückständigen“ und die Landkreise Sümeg, Bicske, Enying im Cluster der "Zurückbleibenden“. Der andere große Teil der fortschreitenden Landkreise umfasst die hauptstädtische Umgebung sowie die von dieser südlich liegenden Landkreise, die Landkreise im Komitat Bács-Kiskun und die östlichen Landkreise im Komitat Tolna. Zu den fortschreitenden Landkreisen gehören auch alle Komitatssitze, weil diese Städte eine begünstigte verwaltungsmäßige Rolle haben, ihre Population groß ist, der Anteil der studierten Leute höher und demgegenüber der Anteil der Arbeitslosen niedriger ist. Diese fortschreitenden Gebiete sind die sich eigenständig entwickelnden Landkreise in den nordöstlichen und südwestlichen Gebieten Ungarns, die ökonomisch benachteiligt sind. Sie gehören zu den sich ökonomisch entwickelnden oder entwickelten Gebieten, die auch zu den besseren Schülerleistungen beitragen.

An der Abbildung 1 kann man auch schlussfolgern, dass die zentralen und westlichen Teile der Region Südtransdanubien zu den Clustern „Unterwegs", "Zurückbleibende“" und „Rückständige" gehören. Von diesen Landkreisen sind nur die Komitatssitze sowie der Landkreis Kaposvár und der Landkreis Pécs ausgenommen. Die schlechtesten Ergebnisse charakterisieren nur die Landkreise des westlichen Teils des Komitats Baranya. Diese Daten hängen mit der schwachen ökonomischen Entwicklung sowie dem hohen Anteil der Population der Roma zusammen.

Wenn wir eine Linie im östlichen Teil des Landes ziehen, wird ein Mosaik von Landkreisen östlich der Linie Szeged-Szolnok-Salgótarján sichtbar. Zwischen dieser Linie und der Grenze können wir alle Typen von Landkreisen entdecken. In der Mehrheit befinden sich hier die Landkreise aus den Clustern „Unterwegs“, „Zurückbleibende“ und „Rückständige“. In diesem Gebiet des Landes gibt es entwickelte Landkreise nur dort, wo es Komitatssitze gibt oder wo die Ökonomie und der Tourismus besser entwickelt sind. Solche Landkreise sind zum Beispiel Kisvárda, Sárospatak oder Hajdúszoboszló. Es ist nicht zufällig, dass die Landkreise in diesem Teil des Landes 


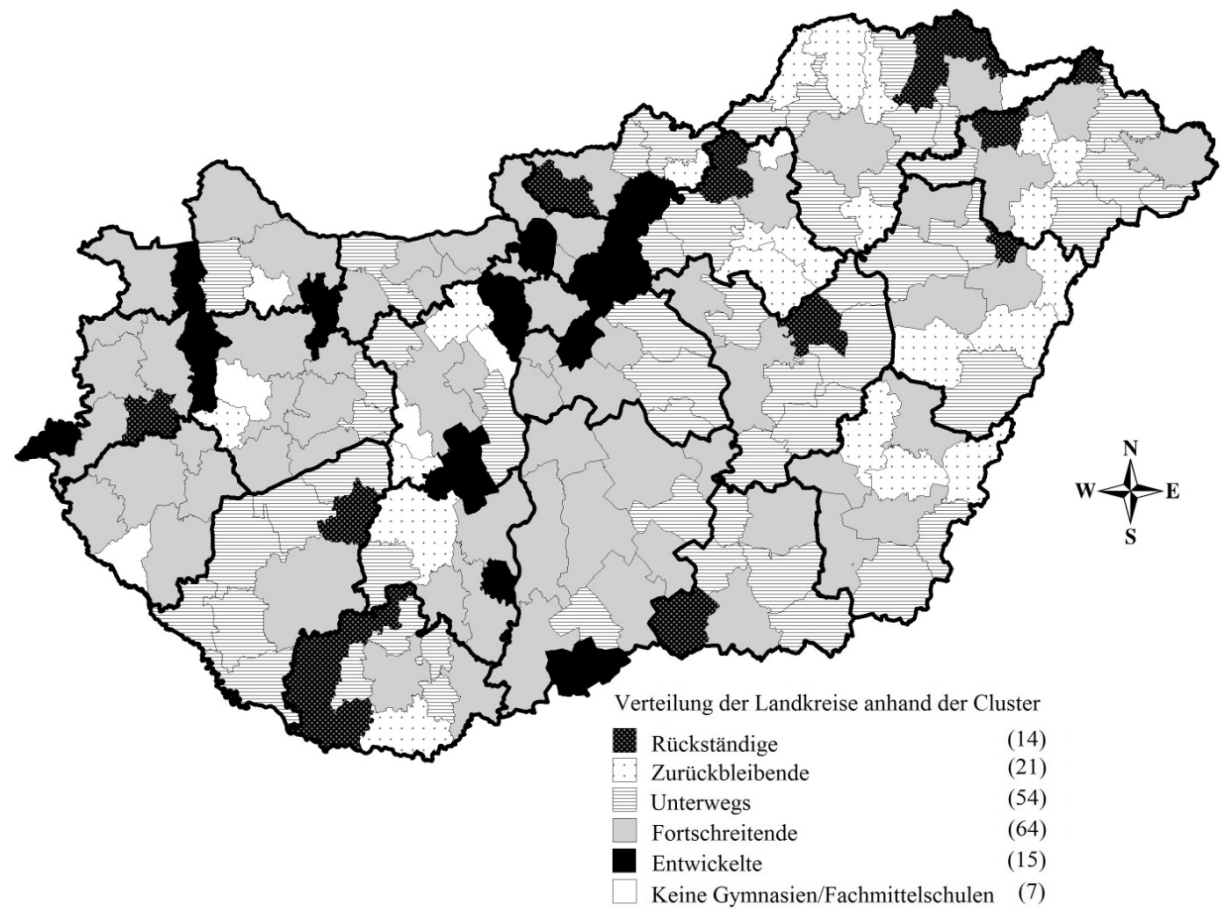

Abbildung 1. Die Cluster aus geografischer / regionaler Sicht $\left(\mathrm{N}_{\mathrm{NKM}}=55156, \mathrm{~N}_{\mathrm{A}}=49732\right)$ Datenquellen: NKM 2013; Abitur 2013-2015.

die schwächsten Leistungen aufweisen, weil der Anteil der studierten Leute hier am niedrigsten, sowie der Anteil der Arbeitslosen am höchsten ist. In diesen Gebieten ist auch der Anteil der Population der Roma sehr hoch. Daher werden diejenigen Landkreise, die jetzt in der Kategorie "Unterwegs" liegen, sich in der Zukunft wahrscheinlich nicht weiter entwickeln.

Am Ende der Untersuchung waren wir daran interessiert, welche Unterschiede sich zwischen den Leistungen der Nationalkompetenzmessung beziehungsweise des Abiturs unter den Gymnasialschülerinnen und Gymnasialschülern und den Schülerinnen und Schülern der Fachmittelschulen ergeben. In der Tabelle 4 befinden sich keine Ergebnisse der Schülerinnen und Schüler der Fachschulen, weil diese Schülerinnen und Schüler im ungarischen Bildungssystem keine Möglichkeit haben, das Abitur als Schulabschluss abzulegen. Wenn diese Schülerinnen und Schüler eine ergänzende Ausbildung absolvieren, dann können sie am Ende das Abitur ablegen. Die anderen zwei Schultypen, das Gymnasium und die Fachmittelschule, zeigen in allen Fällen signifikante Unterschiede in den Ergebnissen der Nationalkompetenzmessung und des Abiturs. Die Gymnasien werden von den Schü- 
lerinnen und Schülern aus Familien mit bedeutend höherem sozioökonomischem Status besucht, deren Eltern einen höheren Bildungsstand und bessere finanzielle Möglichkeiten haben als die Eltern der Schülerinnen und Schüler, die in einer Fachmittelschule lernen. Die Leistungen im Leseverstehen und in der Mathematik zeigen ebenfalls bedeutende Unterschiede, denn die Gymnasialschülerinnen und Gymnasialschüler erlangten fast 170 Punkte mehr in der Nationalkompetenzmessung.

Bei den gemessenen Kompetenzen gibt es auch Unterschiede. Die Ergebnisse in Mathematik sind in beiden Schultypen höher als die Ergebnisse im Leseverstehen. Die Ergebnisse im Abitur zeigen auch ähnliche Abweichungen, weil die Gymnasialschülerinnen und Gymnasialschüler nicht nur in Englisch, sondern auch in Deutsch bessere Leistungen aufweisen. In den Gymnasien in Englisch sowie in Deutsch erreichten die Schülerinnen und Schüler eine starke Vier (gut), die im ungarischen fünfstufigen Notensystem eine gute Note darstellt ${ }^{8}$. In den Fachmittelschulen zeigen die Leistungen in Englisch eine starke Drei (befriedigend), die Leistungen in Deutsch hingegen eine schwache Drei (befriedigend). Diese Ergebnisse bedeuten auch, dass die Schülerinnen und Schüler in beiden Schultypen bessere Englischkenntnisse aufweisen, wobei der Unterschied der Ergebnisse in den Fachmittelschulen größer ist.

Tabelle 4. Die Schülerleistungen in den verschiedenen Schultypen

\begin{tabular}{|l|c|c|c|}
\hline \multicolumn{1}{|c|}{ Veränderlichen } & Gymnasium & Fachmittelschule & Durchschnitt \\
\hline Ergebnisse in der Mathematik & 1802,969 & 1629,953 & 1696,371 \\
\hline Ergebnisse im Leseverstehen & 1778,588 & 1610,123 & 1679,078 \\
\hline Familienhintergrundindex & 0,774 & $-0,044$ & 0,274 \\
\hline Abitur in der Mittelstufe in Englisch & 72,928 & 50,288 & 62,480 \\
\hline Abitur in der Mittelstufe in Deutsch & 69,457 & 45,595 & 57,258 \\
\hline
\end{tabular}

(NNKM= 55156, NA=49732) p=0.000 Datenquellen: NKM 2013; Abitur 2013-2015

Wie auch andere Untersuchungen (z. B. Hartig \& Jude 2008; Csapó et al. 2009) zeigen unsere Ergebnisse, dass die Schülerleistungen in den Gymnasien höher sind. Das kann auch bedeuten, dass die Mehrheit der Schülerinnen und Schüler in den Gymnasien einen höheren gesellschaftlichen Status hat.

${ }^{8}$ Die Noteneinstufung sieht in Ungarn wie folgt aus: 1 - nicht genügend, 2 - genügend, 3 - befriedigend, 4 - gut, 5 - sehr gut. 


\section{FAZIT}

In unserem Artikel analysierten wir die Ergebnisse der Nationalkompetenzmessung 2013 und die Ergebnisse des Abiturs in der Mittelstufe in Englisch und in Deutsch bezüglich der im Jahre 2013 die zehnte Klasse besuchenden Schülerinnen und Schüler. Ziel der Untersuchung war es, die Zusammenhänge zwischen den auf der Landeskreisebene aggregierten Variablen zu erschließen. Als Ergebnis bekamen wir Informationen über die Korrelation der Schülerergebnisse, den Familienhintergrund der Schülerinnen und Schüler sowie den Anteil der Gymnasialschülerinnen und Gymnasialschüler. Diese Angaben analysierten wir in ganz Ungarn, so dass wir auch regionale Unterschiede darstellen konnten.

Unsere Ergebnisse zeigen, dass die Leistungsvariablen in allen Landkreisen eine starke, manchmal eine sehr starke Korrelation haben. Je höher der Familienhintergrundindex in einem Landkreis ist, desto besser sind die Schülerleistungen in der Nationalkompetenzmessung sowie im Abitur in der Mittelstufe. Gleiches gilt auch umgekehrt: In Gebieten, in denen die Schülerleistungen höher sind, kann man auch einen höheren Bildungsstand der Eltern sowie einen höheren Familienstatus feststellen. Man kann weiterhin schlussfolgern, dass die höheren Testergebnisse auch höhere Leistungen in anderen Tests ergeben.

Die fünf Landkreiscluster können die Schülerleistungen gut zeigen, weil die schlechtesten Ergebnisse aller Variablen einen Endpunkt ergeben und die entwickelten Landkreise mit den besten Leistungen am anderen Ende der Skala liegen. Im Mittelpunkt stehen die Landkreise, die sozusagen „unterwegs" sind, und von denen wir noch nicht endgültig wissen, in welche Richtung sie sich weiterentwickeln. Die Landkreise, die eine rückständige Nachbarregion haben, werden in der Zukunft wahrscheinlich auch zurückbleiben. Zugleich werden sich die Landkreise, die eine entwickelte Nachbarregion haben, auch selbst entwickeln (vgl. Enyedi 2000).

Die anhand der fünf Cluster skizzierte Landkarte zeigt damit auch die gespaltene ökonomische Entwicklung des Landes. Zum Beispiel weisen die Hauptstadt und ihre Umgebung, die vom Plattensee nördlich liegenden Gebiete, die Mitte des Landes beziehungsweise die Komitatssitze die besten Ergebnisse auf. Die schwächsten Leistungen charakterisieren Ungarns südwestliche und nordöstliche Gebiete. Hier sind die traditionsreichen Industriegebiete verschwunden oder stillgelegt und auch die landwirtschaftlichen Bedingungen sind ungünstig. So ist es keine Überraschung, dass die gut qualifizierten Arbeitskräfte in diesen Regionen typischerweise abwandern. Das bedeutet auch, dass der Anteil der niedrig qualifizierten Arbeitskräfte dort groß ist, weshalb die Schülerleistungen schlechter sein könnten. Des 
Weiteren ist der Anteil der Population der Roma in diesen Gebieten größer, unter denen ein hoher Bildungsstand selten vorkommt.

Am Ende unseres Artikels arbeiteten wir Informationen über die Leistungsunterschiede in den verschiedenen Schultypen heraus. Man kann feststellen, dass Eltern mit einem höheren Bildungsstand Gymnasien für ihre Kinder bevorzugen, wohingegen Eltern mit einem niedrigeren Bildungsstand lieber Fachmittelschulen für ihre Kinder wählen. Es ist auch sichtbar geworden, dass ein bedeutender Unterschied zwischen den Ergebnissen der verschiedenen Schultypen bei der Nationalkompetenzmessung und dem Abitur zu erkennen ist. Die Gymnasialschülerinnen und Gymnasialschüler zeigen bei den Kompetenztests in Mathematik und beim Abitur in der Mittelstufe in Englisch bessere Leistungen.

Die Ergebnisse der Untersuchung werfen ein allgemeines Bild auf die Leistungen ungarischer Schülerinnen und Schüler und bieten eine feste Basis für andere Mikro-Schulforschungen, die sich auf regionale, gute Praxen oder örtliche Probleme konzentrieren.

\section{LITERATURVERZEICHNIS}

Arató, F. / Varga, A. (2004). Együttmúködés az együttnevelésért. Educatio, 13 (3), 503-508.

Artelt, C. / Schiefele, U. / Schneider, W. / Stanat, P. (2002). Leseleistungen deutscher Schülerinnen und Schüler im internationalen Vergleich (PISA). Zeitschrift für Erziehungswissenschaft, 5 (1), 6-27.

Balázsi, I. / Horváth, Zs. (2010). A közoktatás minősége és eredményessége. In: É. Balázs / M. Kocsis / I. Varga (Hrsg.), Jelentés a magyar közoktatásról 2010 (S. 325-632). Budapest: Oktatáskutató és Fejlesztő Intézet.

Baranyi, B. / Kanalas, I. / Kiss, A. (2006). Perifériatérségek Magyarországon. In: I. Kanalas / A. Kiss (Hrsg.), Perifériaképződés típusai és megjelenési formái Magyarországon (S. 210-233). Kecskemét: MTA RKK Alföldi Tudományos Intézet.

Benito, R. / Alegre, M. A. / Gonzàlez-Balletbò, I. (2014). School segregation and its effects on educational equality and efficiency in 16 OECD comprehensive school systems. Comparative Education Review, 58 (1), 104-134.

Bernstein, B. B. (2003). Class, codes and control. London: Routledge.

Bourdieu, P. (1983). Ökonomisches Kapitel, kulturelles Kapital, soziales Kapital. In: R. Kreckel (Hrsg.), Soziale Ungleichheiten (S. 183-198). Göttingen: o. H.

Coleman, J. S. / Campbell, E. Q. / Hobson, C. J. / McPartland, J. / Mood, A. M. / Weinfeld, F. D. / York, R. L. (1966). Equality of educational opportunity. Washington: U. S. Government Printing Office.

Csapó, B. (2001). A nyelvtanulást és a nyelvtudást befolyásoló tényezők. Iskolakultúra, 11 (8), $25-35$.

Csapó, B. / Molnár, Gy. / Kinyó, L. (2009). A magyar oktatási rendszer szelektivitása a nemzetközi összehasonlító vizsgálatok eredményeinek tükrében. Iskolakultúra, 19 (3-4), 3-13.

Csécsiné Máriás, E. / Hagymássy, T. / Könyvesi, T. (2015): Statisztikai tájékoztató. Oktatási évkönyv 2013/2014. Budapest: Emberi Eróforrások Minisztériuma. 
Denzler, S. / Wolter, S. C. (2010). Wenn das Nächstgelegene die erste Wahl ist. Der Einfluss der geographischen Mobilität der Studierenden auf die Hochschullandschaft Schweiz. Aarau: SKBF.

Einhorn, Á. (2007). Az idegen nyelvi érettségi vizsga reformja. In: I. Vágó (Hrsg.), Fókuszban a nyelvtanulás (S. 73-105). Budapest: Oktatáskutató és Fejlesztő Intézet.

Élő idegen nyelv vizsgakövetelmények (2016). http:/ / www.oktatas.hu/pub_bin/dload/kozokta tas/erettsegi/vizsgakovetelmenyek2017/elo_idegen_nyelv_vk_2017.pdf [Zugriff am: 14.03.2018].

Entorf, H. (2005). PISA-Ergebnisse, sozioökonomischer Status der Eltern und Sprache im Elternhaus. Eine international vergleichende Studie vor dem Hintergrund unterschiedlicher Einwanderungsgesetze. Tertium Comparationis, 11 (2), 134-151.

Enyedi Gy. (2000). Globalizáció és a magyar területi fejlődés. Tér és Társadalom, 14 (1), 1-10.

Ettrich, K.-U. / Krause, R. / Hofer, M. (1996). Der Einfluß familienbezogener Merkmale auf die Schulleistungen ost- und westdeutscher Jugendlicher. Unterrichtswissenschaft, 24 (2), 106-127.

Fehérvári, A. (2015). Társadalmi mobilitás és az iskola. In: A. Varga (Hrsg.), A nevelésszociológia alapjai (S. 183-209). Pécs: Wlislocki Henrik Szakkollégium, PTE BTK NTI Romológia és Neveléstudományi Tanszék, Romológia Kutatóközpont.

Fekete, A. / Hegedû́s, R. / Sebestyén, K. (2016). First-year English and German language teacher majors' profile: From where? Who? Why? And how? In: I. Falus / J. Orgoványi-Gajdos (Hrsg.), New aspects in European teacher education (S. 115-133). Eger: Líceum.

Földes, Cs. (2016). Position und Entwicklungsdynamik des Deutschen als Fremdsprache international - im Blick der angewandten Linguistik. Glottodidactica. An International Journal of Applied Linguistics, 63 (2), 13-38.

Furcsa, L. (2014). Inequalities of education as consequences of grouping arrangements in language classes of disadvantaged children. International Dialogues on Education: Past and Present, 1 (3), 13-21. http://www.ide-journal.org/article/2014-volume-1-number-3-inequali ties-of-education-as-consequences-of-grouping-arrangements-in-language-classes-of-disad vantaged-children/ [Zugriff am: 14.03.2018].

Fürst, D. / Schubert, H. (2001). Regionale Akteursnetzwerke zwischen Bindungen und Optionen. Über die informelle Infrastruktur des Handlungssystems bei der Selbstorganisation von Regionen. Geographische Zeitschrift, 89 (1), 32-51.

Garami, E. (2009a). A humán erőforrás területi különbségei. Az emberi fejlődés indexének hazai alkalmazhatósága. Területi Statisztika, 49 (3), 280-298.

Garami, E. (2009b). A legkiválóbb középiskolák területi különbségei. Educatio, 18 (2), 241-256.

Garami, E. (2014). Kistérségi jellemzők és az oktatás eredményessége. Educatio, 23 (3), 424-437.

GER (2002): Közös Európai Referenciakeret. Európa Tanács. http://www.nyak.hu/nyat/doc/ KER_2002/KER_2002.ZIP [Zugriff am: 14.03.2018].

Gesetz LXV/2015. törvény a nemzeti köznevelésról szóló 2011. évi CXC. törvény módosításáról.

Gogolin, I. (2014). Stichwort: Entwicklung sprachlicher Fähigkeiten von Kindern und Jugendlichen im Bildungskontext. Zeitschrift für Erziehungswissenschaft, 17 (3), 407-431.

Hartig, J. / Jude, N. (2008). Sprachkompetenzen von Mädchen und Jungen. In: E. Klieme (Hrsg.), Unterricht und Kompetenzerwerb in Deutsch und Englisch. Ergebnisse der DESI-Studie (S. 202-207). Weinheim: Beltz.

Hegedûs, R. (2016). A LeaRn index és a tanulói teljesítmény területi összefüggése. Educatio, 25 (2), 268-277.

Henry, D. L. / Baltes, B. / Nistor, N. (2015). Social mobility through mathematics proficiency for English language learners. Journal of Social Change, 7 (1), 94-102.

Hesse, H.-G. / Göbel, K. / Hartig, J. (2008). Sprachliche Kompetenzen von mehrsprachigen Jugendlichen und Jugendlichen nicht-deutscher Erstsprache. In: E. Klieme (Hrsg.), Unter- 
richt und Kompetenzerwerb in Deutsch und Englisch. Ergebnisse der DESI-Studie (S. 208-230). Weinheim: Beltz.

Imre, A. (2002). Az iskolai hátrány összetevői. Educatio, 11 (1), 63-72.

Kaikkonen, P. (1993). Fremdsprachenlernen - ein individueller, kulturbezogener Prozess einige Beobachtungen im Rahmen eines erlebten Kultur- und Landeskunde betonenden Unterrichtsversuches. Unterrichtswissenschaft, 21 (1), 2-20.

Kétszintú érettségi eredmények (2016). https://www.ketszintu.hu/publicstat.php [Zugriff am: 14.03.2018].

Kozma, T. (1996). Településhálózat és iskolarendszer. Educatio, 5 (2), 248-249.

Linberg, T. / Wenz, S. E. (2017). Ausmaß und Verteilung sozioökonomischer und migrationsspezifischer Ungleichheiten im Sprachstand fünfjähriger Kindergartenkinder. Journal for Educational Research Online / Journal für Bildungsforschung Online, 9 (1), 77-98.

Neumann, M. / Nagy, G. / Trautwein, U. / Lüdtke, O. (2009). Vergleichbarkeit von Abiturleistungen Leistungs- und Bewertungsunterschiede zwischen Hamburger und Baden-Württemberger Abiturienten und die Rolle zentraler Abiturprüfungen. Zeitschrift für Erziehungswissenschaft, 12 (4), 691-714.

Niedrig, H. (2002). Strategien des Umgangs mit sprachlicher Vielfalt. Analyse bildungspolitischer und konzeptioneller Ansätze. Tertium Comparationis, 8 (1), 1-13.

Nikolov, M. (2003). Angolul és németül tanuló diákok nyelvtanulási attitúdje és motivációja. Iskolakultúra, 13 (8), 61-73.

Nikolov, M. / Vígh, T. (2012). Az idegen nyelvek tanulásának eredményessége. In: B. Csapó (Hrsg.), Mérlegen a magyar iskola (S. 241-288). Budapest: Nemzeti Tankönyvkiadó.

Óhidy, A. (2007). Das ungarische Bildungssystem. In: A. Óhidy / E. Terhart / J. Zsolnai (Hrsg.), Lehrerbild und Lehrerbildung. Praxis und Perspektiven der Lehrerausbildung in Deutschland und Ungarn (S. 67-95). Wiesbaden: Verlag für Sozialwissenschaften.

Oswald, H. / Krappmann, L. (2004). Soziale Ungleichheit in der Schulklasse und Schulerfolg. Eine Untersuchung in dritten und fünften Klassen Berliner Grundschulen. Zeitschrift für Erziehungswissenschaft, 7 (4), 479-496.

Petneki, K. (2002). Az idegen nyelv tanításának helyzete és fejlesztési feladatai. Új Pedagógiai Szemle, 52 (7-8), 147-160.

Polónyi, I. (2013). Az aranykor vége - bezárnak-e a papírgyárak? Budapest: Gondolat Kiadó.

Pop-Eleches, C. / Urquiola, M. (2013). Going to a better school: Effects and behavioral responses. American Economic Review, 103 (4), 1289-1324.

Rolff, H.-G. / Leucht, M. / Rösner, E. (2008). Sozialer und familialer Hintergrund. In: E. Klieme (Hrsg.), Unterricht und Kompetenzerwerb in Deutsch und Englisch. Ergebnisse der DESI-Studie (S. 283-300). Weinheim: Beltz.

Scheiner, J. (2006). Wohnen und Aktionsraum: Welche Rolle spielen Lebensstil, Lebenslage und Raumstruktur? Geographische Zeitschrift, 94 (1), 43-62.

Schultz, T. W. (1998). Beruházás az emberi tókébe. In: Gy. Lengyel / Z. Szántó (Hrsg.), Tőkefajták. A társadalmi és kulturális erőforrások szociológiája (S. 45-69). Budapest: Aula.

Süli-Zakar, I. (2003). A magyar területfejlesztés az EU regionális politikájának tükrében. Az Észak-Alföldi régió fejlesztési dokumentumai az EU csatlakozás tükrében. In: I. Süli-Zakar (Hrsg.), Társadalom földrajz és területfejlesztés II (S. 639-666). Debrecen: Debreceni Egyetem Kossuth Egyetemi Kiadója.

Szczesny, M. / Watermann, R. (2009). Differenzielle Einflüsse von Familie und Schulform auf Leseleistung und soziale Kompetenzen. Journal for Educational Research Online / Journal für Bildungsforschung Online, 3 (1), 168-193. 
Széll, K. (2015). Az iskolai eredményesség a hátrányos helyzet tükrében. Educatio, 24 (1), 140-147.

Szirmai, H. (2003). A matematikai és a nyelvi képesség közötti összefüggés vizsgálata. Új Pedagógiai Szemle, 53 (5), 24-37.

Torgyik, J. (2015). Multikulturalizmus, interkulturális nevelés. In: A. Varga (Hrsg.), A nevelésszociloógia alapjai (S. 161-181). Pécs: Wlislocki Henrik Szakkollégium, PTE BTK NTI Romológia és Neveléstudományi Tanszék, Romológia Kutatóközpont.

Verordnung Nr. 100/1997. (VI. 13.) Korm. rendelet az érettségi vizsga szabályzatának kiadásáról.

Verordnung Nr. 16/2000. (II. 11.) Korm. rendelet az érettségi vizsga szabályzatának kiadásáról szóló 100/1997. (VI. 13.) Korm. rendelet módosításáról.

Veroszta, Zs. (2010). A munkaerő-piaci sikeresség dimenziói frissdiplomások körében. In: O. Garai / T. Horváth / L. Kiss / L. Szép / Zs. Veroszta (Hrsg.), Diplomás pályakövetés IV. Frissdiplomások 2010 (S. 11-36). Budapest: Educatio Társadalmi Szolgáltató Nonprofit Kft.

Received: 5.04.2018; revised: 1.07.2019 


\author{
EMILIA KUBICKA \\ Uniwersytet Mikołaja Kopernika w Toruniu \\ ekubicka@umk.pl \\ ORCID: 0000-0002-4026-5420
}

\title{
So-called dictionary equivalents confronted with parallel corpora (and the consequences for bilingual lexicography) ${ }^{*}$
}

\begin{abstract}
The study considers the question of whether (and how) bilingual dictionaries may be improved. The information presented in dictionaries has been confronted with textual reality (i.e., with examples of actual translations), based on the German expression fassungslos and its Polish equivalents in parallel texts. The author assumes that bilingual dictionaries are mainly used by language learners, while professional translators may consider them as one of many possible sources. In teaching, multiplying the possible equivalents or suggesting ad hoc solutions is generally not recommended. Despite the attempts at objectivizing lexicographic descriptions, which are made possible by using language corpora, it often turns out that the decisions made by dictionary authors are (and need to be) arbitrary.
\end{abstract}

KEYWORDS: Translation studies, bilingual lexicography, dictionary equivalents, parallel corpus.

\section{PRELIMINARY REMARKS}

Bilingual dictionaries are among the most popular and most commonly used types of dictionaries, particularly when it comes to learning foreign languages. At the early stages of the learning process, their content is rarely being questioned. This is also the case when they are used by non-specialists evaluating the quality of translations. However, scholars dealing with trans-

* The paper was written with the support of DAAD (The German Academic Exchange Service), within the framework of Re-invitation Program for Former Scholarship Holders (February-March 2018). 